\title{
Quartiere kultivieren Quartierbilder - Quartierbilder kultivieren Quartiere ${ }^{1}$
}

\section{Einleitung}

Was kommt Ihnen in den Sinn, wenn Sie an Ihr Wohnquartier denken? Kommen Ihnen ganz bestimmte Dinge, Orte oder Ereignisse in den Sinn? Denken Sie vielleicht an Ihre Nachbarn, deren Häuser oder an Einkaufsläden, die Sie nutzen. Sehen Sie das alles, wie es heute ist oder wie es früher war? Kurz: Wie sehen Sie eigentlich Ihr Quartier? Und: Wie sehen wohl Ihre Nachbarn, andere Quartierbewohner oder Bekannte von Ihnen, die in einem anderen Quartier wohnen, ihr Quartier? Das sind die Fragen, denen wir in diesem Beitrag nachgehen wollen.

Im Vordergrund steht damit nicht die Bearbeitung von Fragen nach räumlicher Orientierung und positiver Identifikation mit einer Stadt, wie sie sich hauptsächlich in der Tradition des Städteplaners Kevin LYNCH (1960) herausentwickelt hat. Unser Interesse gilt deshalb weder Problemen der "Lesbarkeit» noch dem Aufbau kognitiver Karten von Städten. Im vorliegenden Beitrag interessiert vor allem die subjektive Bedeutung städtischer Umwelten. Die Beschäftigung mit dieser bedeutungsbezogenen Umweltrepräsentation ist zwar bei LYNCH durchaus angelegt. Es wäre aber eine unangemessene Vereinfachung, würde man die Bedeutung städtischer Umwelten, in der sich Menschen aufhalten und handeln, ausschließlich - wie das in der Regel die kognitiven Ansätze der Verhaltensgeographie tun - im Zusammenhang mit ihrem räumlichen Verhalten behandeln (GOTTDIENER und LAGOPOULOS 1986; SCHNEIDER 1990).

Beginnen wollen wir unseren Beitrag mit einem kurzen Abriß über die Forschungen zur subjektiven Bedeutung von Umwelt, um schließlich einen theoretisch weiterführenden Beitrag zu leisten, zumal sich in diesem Forschungsbereich - im Unterschied zur «kognitiven Kartenforschung" - bisher erst rudimentäre theoretische Ansätze finden lassen. Zentraler Gedanke dabei wird sein, daß menschliche Erfahrungen und Vorstellungen im Umgang mit dem Wohnquartier u. a. in Form kognitiver Bilder (images) repräsentiert sind, die ihrerseits die Grundlage künftiger Erfahrungs- und Vorstellungsbildungen im Umgang mit dem Quartier darstellen.

Damit wird deutlich, daß die Quartierumwelt des Menschen immer schon als kultürliche Umwelt zu betrachten ist, die durch die Quartierbilder ihrer Bewohner «kultiviert» wird und ihrerseits ihre Bewohner - und deren Bilder vom Quartier - «kultiviert». Diese Kultivationsthese wurzelt in den kultursoziologischen und -psychologi- schen Arbeiten von Georg sIMMEL (1908) und deren Weiterentwicklungen durch ROCHBERG-HALTON (1986) und FUHRER (im Druck). Diesen konzeptuellen Darlegungen schließt sich eine empirische Studie an, in deren Rahmen untersucht wurde, nach welchen Bedeutungsdimensionen Quartierbewohner sowohl ihr eigenes als auch ein anderes Wohnquartier "sehen", d. h. kognitiv repräsentieren.

\section{Bedeutungskomponenten der Umwelt}

In der Literatur über subjektive Bedeutung von Umwelt lassen sich kognitive, emotional-evaluative, behaviorale sowie symbolische Komponenten voneinander unterscheiden (SCHNEIDER 1990).

Kognitive Komponente: Das methodische Standardverfahren besteht darin, fotografisch repräsentierte Umweltausschnitte (städtische Szenen, Wohngegenden, Bauten, Landschaften usw.) mittels paarweiser Beurteilung, Rangreihenbildung oder mittels freien Sortierens auf ihre Ähnlichkeit hin einschätzen zu lassen. Diese Ähnlichkeitsurteile werden darauf in der Regel dimensions(multidimensionale Skalierung; MDS) oder clusteranalytisch auf einfachere Strukturen zu reduzieren versucht. Die Ergebnisse fallen dabei üblicherweise sehr unterschiedlich aus. Dies mag einerseits mit den weitgehend fehlenden theoretischen Grundlagen (einer Ergebnisinterpretation stehen unbegrenzt viele Möglichkeiten offen) und andererseits mit der Auswahl der Fotos zusammenhängen. Weil nämlich die Fotos nicht theoriegeleitet ausgewählt werden können, üben sie ihrerseits einen schwer kontrollierbaren Einfluß aus. Schließlich gilt es noch zu bedenken, daß auch im Falle inhaltlich sinnvoller Lösungen nicht ohne weiteres auf eine entsprechende mentale Repräsentation geschlossen werden kann (WARD und RUSSELL 1982).

Emotional-evaluative Komponente: Die übliche Art, emotionale Bedeutungen von Bauten, Quartieren, Städten, Siedlungen, Landschaften usw. zu erfassen, ist das Semantische Differential (SD) oder Polaritätsprofil. Darin läßt sich auf leicht verständlichen, kontinuierlichen, bi-

Urs Fuhrer, PD Dr. phil. I, Florian G. Kaiser, lic. phil. I, Manuela Marxer, cand. phil. I, Psychologisches Institut der Universität Bern, Laupenstraße 4, CH-3008 Bern 
polaren Skalen gegensätzlichen Umweltbedeutungen mehr oder weniger zustimmen. Problematisch ist im wesentlichen die Interpretation der bipolaren Aussagen. Nicht zuletzt deshalb ist in der Umweltpsychologie eine methodenkritische Diskussion dieses Verfahrens entstanden (z. B. RUSSELL, WARD und PRATT 1981), worin auf Probleme der Bedeutungskonfundierung sowie auf den hier ebenfalls häufig fehlenden Theoriebezug verwiesen wird.

Behaviorale Komponente: Die Bedeutung räumlicher Umwelt läßt sich aber auch über individuelle und/oder kollektive Verhaltensweisen, die ein Ort ermöglicht, festlegen. So können einzelne Handlungen räumlich lokalisiert werden, was beispielsweise Konzepte wie «gelebter Raum» (MUCHOW und MUCHOW 1935) und "Aktionsraum» (FRIEDRICHS 1977) beinhalten. Darin zeigen sich die Ortsspezifitäten einzelner Verhaltensweisen. Demgegenüber kann aber auch die Verhaltensvarianz, die ein einzelner Ort zuläßt, betrachtet werden, um diesen Ort über Verhaltensweisen zu definieren, wie es für den von BARKER und Mitarbeitern entwickelten Behavior-SettingAnsatz kennzeichnend ist (BARKER 1968; FUHRER 1990). Dadurch zeigen sich die Verhaltensspezifitäten einzelner Orte.

Symbolische Komponente: Sowohl kognitive als auch emotional-evaluative sowie behaviorale Bedeutungen der Umwelt beinhalten alle gleichermaßen, daß Umwelten und ihre Dinge nicht nur objektive, als solche vorfindbare, sondern auch "über sich hinausweisende», für etwas anderes stehende Gegebenheiten sind. In diesem Sinn sehen auch GOTTDIENER und LAGOPOULOS (1986) ihren sozio-semiotischen Ansatz. Auch sie rücken den Prozeß der Symbolgenese über die Mensch-Umwelt-Beziehung in den Blickpunkt ihres Interesses und meinen, daß sich Orte und ihre Dinge dadurch definieren, daß sie für etwas stehen und daß sie im Bewußtsein ihrer «Interpreten» ein Zeichen erzeugen. Als Konventionen, die sich im wiederholten Gebrauch herausbilden, verweisen diese Zeichensymbole auf ihre Orte, ihre Dinge und geben ihnen Bedeutungen. Der Bezug zwischen Zeichen und Bezeichnetem ist letztlich aber arbiträr, wodurch auch erklärt ist, weshalb Symbole individuums- und kulturabhängig sind (BOESCH 1980). So kann eine Trabantensiedlung für die einen Häßlichkeit, Benachteiligung und soziale Minderwertigkeit, für die anderen Sauberkeit, Gemütlichkeit und Heimat symbolisieren bzw. bedeuten (WEICHHARDT 1989). Die symbolische Bedeutung eines Ortes oder Dinges läßt sich folgerichtig auch erst mit Hilfe der persönlichen Lebensgeschichte des Bedeutungsverleihers vollständig verstehen.

Beispiele solcher symbolischer Veräußerungen psychischer Strukturen in Orten sind die stadtbezogene Identität, die lokale und die regionale Identität (WEICHHARDT 1990), die sich alle im Konzept Ortsidentität (PROSHAN. SKY, FABIAN und KAMINOFF 1983) zusammenfassen lassen. Damit werden Orte zu Symbolen des Selbst und strukturieren ihrerseits die Wahrnehmungs- und Handlungserfahrungen von Individuen (und Gruppen) in viel- fältiger Weise mit (STOKOLS 1981; GOTTDIENER und LAGOPOULos 1986). Zieht man zudem in Betracht, daß die Ortsidentität eine der Grundlagen emotionaler Bindung an Orte bildet (FUHRER und KAISER 1992a; 1992b), wird zusätzlich zum rein bewußt-kognitiven auch der emotionale Symbolgehalt evident.

\section{Kultivation als Rahmenkonzept}

Für eine im engeren Sinne kulturpsychologische Betrachtung der Bedeutung von Umwelt hat immer noch jene von LEWIN (1917) in seinem Aufsatz "Kriegslandschaft» formulierte und später u. a. von BOESCH (1980) elaborierte Feststellung Gültigkeit, wonach Menschen sowohl natürliche als auch gestaltete Umwelt - wie es z. B. Wohnumwelten, Stadtteile, Dörfer, Städte, Landschaften usw. sind - nicht allein passiv erdulden. Das heißt, Umweltbedeutungen hängen nicht nur von ihren sachlichen, objektiven Qualitäten ab. So unterscheiden sich Trabantenstädte eben nicht nur physisch-morphologisch von historisch gewachsenen Stadtquartieren oder Einfamilienhaussiedlungen, sondern auch in der Lebensart und den Denk- und Handlungsweisen ihrer Bewohner. Wie auch immer die Bedeutung einer Landschaft, eines Ortes oder eines Quartiers wahrgenommen wird, letztlich sind es immer Bedeutungen der Umwelt fuir einen darin Handelnden: So unterscheidet sich eben die Quartierbedeutung eines im Quartier aufgewachsenen, täglich seine Einkäufe besorgenden, seine Freunde besuchenden Quartierbewohners deutlich sowohl von den Quartierbedeutungen desjenigen, der auf seinem täglichen Arbeitsweg nur durchfährt, als auch von jenem, der das Quartier «nur vom Hörensagen" kennt. Das Quartier als Gegebenheit ist folglich nicht in erster Linie wegen seiner objektiven, sondern vor allem wegen seiner psychologischen, d. h. wegen seiner erlebens- und handlungsbezogenen Eigenschaften bedeutsam.

Zwei Arten von Bedeutungen sind grundsätzlich analytisch unterscheidbar: Zum einen nehmen Menschen Umwelt immer schon strukturiert und damit bedeutsam wahr. Eine solche Strukturierung entspricht den früher gemachten Handlungserfahrungen, womit sich aber streng genommen Handlungserfahrungen selbst diejenigen kognitiven und mentalen Strukturen schaffen, die wiederum Handlungserfahrungen erst möglich machen. Zum andern wird Umwelt dadurch bedeutsam, daß man sie in einer bestimmten Weise nutzt. Das bedeutet, daß die Art und Weise, wie Menschen ihre Umwelt gestalten, diese Umwelt physisch (mit)konstituiert. So schlagen sich beispielsweise in Wohnquartieren die Handlungsspuren menschlicher Tätigkeiten nieder und stellen damit ihrerseits Möglichkeiten und auch Beschränkungen individuellen und sozialen Handelns dar (FUHRER im Druck). Und weil sich im Gestalteten Handlungserfahrungen ebenso niederschlagen wie im mentalen (inneren) Gedächtnis, liegt es nahe, Gebautes und Gestaltetes mit seinen Dingen als externes Gedächtnis zu begreifen, 
das für einen selbst wie für andere einen überdauernden, kollektiven Rahmen für das Handeln setzt (LANG 1992). Dieses externe Gedächtnis stellt somit, weil es auch für andere Menschen zugänglich und für diese ebenfalls benutzbar ist, ein überindividuelles, soziales und kulturelles Gedächtnis dar.

Umwelten, in die Menschen eingebunden sind, sind demzufolge immer schon kultürliche Umwelten, weil Menschen sie einerseits nach ihren eigenen kulturellen Schemata wahrnehmen und bewerten, andererseits auch behandeln (BOESCH 1980). Mit diesen Überlegungen möchten wir überleiten zur Kultivierungsthese (vgl. Abb. 1). Diese besagt, daß Orte einerseits die in ihnen lebenden Menschen und daß andererseits diese Menschen ihre Orte kultivieren. Um diesen Gedanken zu verdeutlichen, kann die von BOESCH (1980) verwendete Kurzformel von der Objektivierung des Subjektiven und der Subjektivierung des Objektiven als Wegweiser dienen. Dabei kann auf der einen Seite zwischen einer objektivierenden Vergegenständlichung (z. B. der Bau von Häusern, Personalisierung von Territorien) und einer objektivierenden Aneignung (z. B. die Orientierung an den Gefährdungsmomenten einer Quartierstraße; Akkommodation im Sinne von PIAGET 1973) unterschieden werden (vgl. OERTER 1983). Auf der anderen Seite kann zwischen einer subjektivierenden Vergegenständlichung (z. B. die Mitgestaltung der Quartierbewohner bei der Planung einer Wohnstraße) und einer subjektivierenden Aneignung (z. B. das Erlernen bestimmter Wege; Assimilation im
Sinne von PIAGET 1973) differenziert werden. In diesem Sinne meint Objektivierung die Kultivation «nach außen", Subjektivierung die Kultivation "nach innen" (FUHRER 1992). Nach außen gerichtete Kultivation manifestiert sich in Handlungsspuren einer Umwelt, die als externes Gedächtnis fungieren und zur Regulation von sozialen Beziehungen im Quartier sowie zur Entwicklung persönlicher und kollektiver Identitäten beitragen können (FUHRER im Druck; FUHRER und KAISER 1992b). Folgerichtig können Handlungsspuren also auch andere beeinflussen, sofern diese Spuren allgemein zugänglich sind (vgl. FUHRER im Druck). Nach innen gerichtete Kultivation manifestiert sich in mentalen, intrapersonalen Strukturen oder Schemata, die der strukturierten Wahrnehmung der Umwelt dienen und dieser damit Bedeutung verleihen.

Abb. 1 zeigt, daß Handlungen im Sinne einer nach außen gerichteten Kultivation subjektive, intrapersonale Vorstellungen in Objektives - für andere Erfahrbares - transformieren. Diese objektiven Handlungsspuren werden ihrerseits über Wahrnehmungsprozesse - im Sinne einer nach innen gerichteten Kultivation - wieder in Intrapersonales, Subjektives zurückgeführt. Diese Subjektivierung der Umwelt schlägt sich u. a. in Form von individuellen oder sozial geteilten Vorstellungen und Bedeutungen (als soziale Repräsentationen; vgl. v. CRANACH 1991) nieder; Bedeutungen, die sich aus der Interaktion der Bewohner mit ihren Umwelten entwickeln und wandeln.

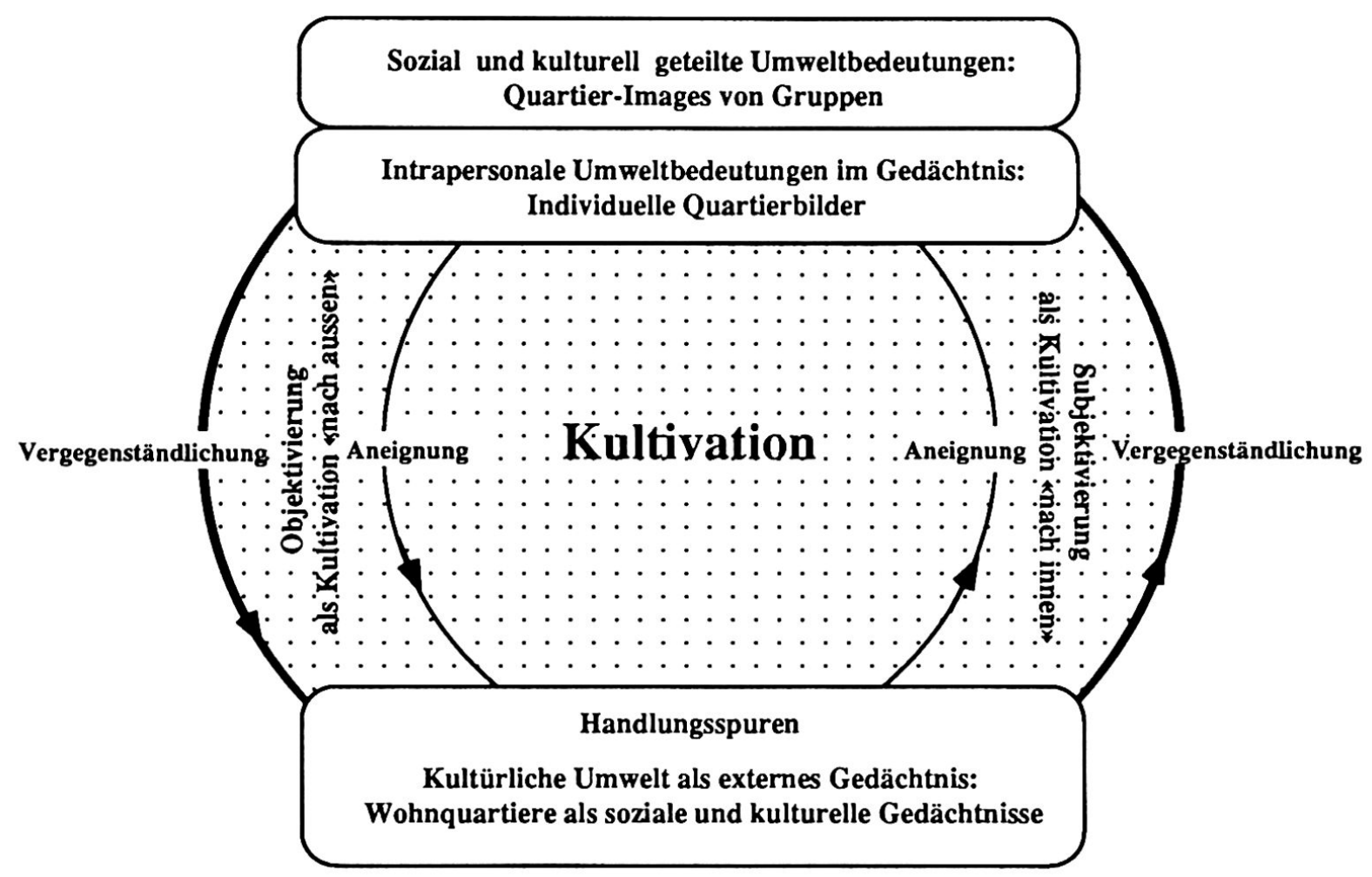

Abb.1 Schematische Darstellung individueller und überindividueller Kultivation in der Mensch-Umwelt-Beziehung. 
Dieser individuelle Kultivationskreis läßt sich nun aber auch in einen weiteren, überindividuellen Kultivations rahmen stellen. Analog der Auffassung von BOESCH (1991), in der der Individualebene mit ihrem individuellen Handlungsfeld eine überindividuelle Kollektivebene mit einem kulturellen Handlungsfeld zugeordnet wird. Vergleichbar damit entwickeln so beispielsweise auch Bewohner eines Quartiers aufgrund ihrer individuellen Ortsbilder bzw. Quartierbilder durch Kommunikation mit anderen überindividuelle Orts- und Quartierbilder im Sinne von RELPH (1976), die mit zunehmender Wohndauer an Ähnlichkeit gewinnen und damit nicht mehr allein individuell gültig sind. RELPH (1976) verwendet für diese sozialisierten Ortsbilder den Begriff «image», um damit die primär überindividuelle Perspektive dieser Vorstellungsbilder zu verdeutlichen. Das Ortsimage manifestiert sich wiederum in den Handlungsspuren der Bewohner und vermittelt so die sozial geteilte Bedeutung des Quartiers weiter. Dadurch wird nun aber das Quartier erst zur überindividuellen - und damit im eigentlichen Sinn zur kultürlichen Umwelt, zum sozialen und kulturellen Gedächtnis, worin die Handlungserfahrungen von Individuen und ganzen Gruppen gespeichert sind.

Beeinflussen die überindividuell bedeutsamen Vorstellungsbilder über die objektivierende Vergegenständlichung oder Aneignung die Umwelt, so beeinflussen die damit verbundenen kollektiven Handlungsspuren auch die subjektivierende Aneignung und Vergegenständlichung in Form intrapersonaler Vorstellungsbilder. Auf diese Weise bestimmen die überindividuellen Bilder, wie sie von vielen sozial geteilt werden, eben auch die individuellen Bilder mit (HART 1987). Empirische Studien zeigen denn auch, daß Ortsimages weitgehend sozialisiert sind (WALTHER 1988), was von LYNCH (1960) und vielen Städteplanern - relativ zur Berücksichtigung von Merkmalen der Lesbarkeit - noch zu wenig mitberücksichtigt worden ist. Damit wird nun aber verständlich, warum das Image, das ein Quartier für Außenstehende hat, auch auf seine Bewohner "abfärbt» (LALLı 1989), und warum bauliche Veränderungen in einem Quartier oder die Präsenz fremder und unbekannter Personen das sozial geteilte Ortsimage verändern können (WALTHER 1988).

Mit dem Kultivationsansatz versuchen wir, die strikte Trennung von Mensch und Umwelt aufzugeben. Denn jeder Mensch ist unauflösbar mit seiner physischen und sozialen Umwelt verbunden: Nach innen und nach außen gerichtete Kultivation läßt sich dabei zwar analytisch, nicht aber praktisch unterscheiden. Über die Kultivation setzt sich das Individuum in einen konkreten, immer schon bedeutungsvollen Bezug zur Welt. Quartiere als kultürliche Umwelten bieten so einerseits Handlungsmöglichkeiten an, andererseits stellen sie auch Handlungsbedingungen, denen die Objektivierungen und Subjektivierungen unterworfen sind. In diesem Sinn ist Kultivation ein aktiver - nach innen wie nach außen gerichteter - Strukturbildungsprozeß, welcher das individuelle Handeln, Wahrnehmen und Denken beeinflußt.

\section{Empirische Untersuchung in zwei Quartieren der Stadt Bern}

\subsection{Methoden}

Versuchspersonen - Untersuchungsorte: Die Untersuchungsstichprobe setzt sich aus 50 Personen (Vpn) aus zwei Quartieren der Stadt Bern zusammen. ${ }^{2}$ Bei den beiden Quartieren handelt es sich um das zentrumsnahe Länggassequartier sowie um das zentrumsfernere Bümpliz. Die Personen wurden per Zufall aus einem Panel von 543 ausgewählt. Insgesamt sind 27 weibliche und 23 männliche Vpn. Der Altersrange liegt zwischen 24 und 85 Jahren (Median $=46$ Jahre).

Versuchsmaterial: Mit Hilfe fotografischen Quellenmaterials (z. B. Quartierinventar, historische Überblicksdarstellungen) und der Beratung zweier "Quartierexperten» (ehemalige Vorstandsmitglieder der lokalen Quartiervereine) wurden je 40 Fotos angefertigt, die die beiden Quartiere in einer repräsentativen Weise wiederzugeben vermögen.

Versuchsdurchführung:Im Hinblick auf die Durchführung einer nichtmetrischen Multidimensionalen Skalierung (MDS) wurde zur Datenerhebung die Methode des "Freien Sortierens" gewählt (KUEHNE 1976). Dazu wurden den Vpn nacheinander je 40 Fotos des eigenen und des fremden Quartiers vorgelegt. Die Vpn hatten diese Fotos so zu gruppieren, daß jeweils «ähnliche» Fotos denselben Gruppen zugeordnet werden mußten. Die Anzahl und Größe der von den Vpn gebildeten Gruppen war beliebig. Gleichzeitig wurde die Gruppierung der Fotos durch die Vpn verbal kommentiert. Die Kommentare wurden auf Tonband aufgezeichnet und anschließend transkribiert.

\subsection{Ergebnisse E $^{3}$ - Diskussion}

In einem ersten Schritt versuchen wir, die überindividuellen Bedeutungsdimensionen, auf denen die entsprechenden Quartierbilder im wesentlichen beruhen und die die Ähnlichkeitsurteile letztlich bestimmen, für beide Quartiere getrennt mittels MDS festzustellen. In einem zweiten Schritt wenden wir uns daraufhin den individuellen Quartierbildern zu, und zwar in Form zweier untypischer Vertreter der überindividuellen Quartierbeurteilung. Dazu werten wir die verbalen Kommentare dieser Vpn aus.

\subsubsection{Zwei überindividuelle Bedeutungsdimensionen von Quartieren: Urbanität und Komplexität}

Für beide Quartiere konnten unabhängig voneinander dieselben zwei Bedeutungsdimensionen als Grundlage der Ähnlichkeitsurteile der Fotos festgestellt werden, wobei die zweidimensionale Lösung im Fall des Länggassequartiers $18 \%(n=49)$, im Fall von Bümpliz $16 \%(n=50)$ der Varianz zu erklären vermag. Offenbar sind im vorliegenden Fall für die Beurteilung beider Quartiere nicht allein quartier-, sondern stadt- oder gar regionstypische 
Bedeutungen wesentlich. Es zeigen sich aber zumindest Ausprägungsunterschiede in diesen - dem überindividuellen Quartierbild zugrunde liegenden - Bedeutungsdimensionen, die im Sinne der Kultivationsidee verstanden werden können. Wenn nämlich Länggassebewohner beide Quartiere in beide Dimensionen stärker ausgeprägt erfahren $(\mathrm{F}=60.6$; df: $4 / 45 ; \mathrm{p}<.001)$, und zwar sowohl das eigene $(F=204.7 ; \mathrm{df}: 1 / 48 ; \mathrm{p}<.001) ; \mathrm{F}=93.6$; df: $1 / 48 ; \mathrm{p}<.001$ ) wie auch das fremde Quartier (Bümpliz) $(F=92.1 ; d f: 1 / 48 ; p<.001) ; F=204.6 ; d f: 1 / 48$; $\mathrm{p}<.001$ ), so scheinen die Wohnerfahrungen im eigenen Quartier nicht spurlos an diesen überindividuellen Dimensionen vorbeigegangen zu sein. Die Prozentanteile erklärter Varianz machen u. a. deutlich, wie groß der individuelle neben dem hier dargestellten überindividuellen Anteil bei der Ähnlichkeitsbeurteilung in etwa sein dürfte. Daraus läßt sich schließen, daß das Sortieren der Fotos und damit das Quartierbild nicht nur von sozial geteilten, überindividuellen, sondern ebenso von individuellen Quartierbildern geleitet wird; ein Resultat, das mit den Ergebnissen von WALTHER (1988) oder von WEICHHARDT (1989) übereinstimmt. Doch wenden wir uns zunächst der inhaltlichen Bestimmung der Bedeutungsdimensionen zu.

Die erste unipolare Dimension kann als «Urbanität» bezeichnet werden. ${ }^{4}$ Darin zeigt sich, daß eine klare Trennung zwischen "Naturfotos» und "Urbanfotos» vorgenommen wurde. Letztere beinhalten vor allem von Menschen gestaltete räumlich-soziale Umwelten im Sinne von "behavior settings" (BARKER 1968). Sie werden mit Tätigkeiten wie Wohnen, Einkaufen, Zur-Schule-Gehen oder mit Arbeiten in Verbindung gebracht. Naturfotos hingegen werden eher mit Erholungs- und Freizeitaktivitäten verknüpft. Dadurch wird deutlich, daß die Urbanität das potentielle Handlungsangebot zumindest mitbeinhaltet.

Die zweite unipolare Dimension kann als «Komplexität» der Bauten bezeichnet werden. Auf dieser Dimension werden die Naturfotos neutral, das heißt um den Nullpunkt, beurteilt. Die urbanen Fotos hingegen variieren in bezug auf ihre physische Komplexität. Einem eher "eintönigen» steht ein eher "vielfältiger» Pol gegenüber. Was dabei auffällt, ist die gleichzeitige strikte Trennung von jüngeren und älteren Bauten. Diese Dimension repräsentiert also einerseits historische und andererseits physische Komplexitätsaspekte der abgebildeten Gebäude. Ebenso wie bei der Urbanitätsdimension kommt hier ebenfalls den Handlungsmöglichkeiten wesentliches Gewicht zu. Diese Interpretation wird auch durch die verbalen Kommentare der Vpn unterstützt. Ältere Bauten werden darin als vielfältiger und interessanter sowohl im Hinblick auf ihr physisches Erscheinungsbild als auch hinsichtlich ihrer Handlungsmöglichkeiten wahrgenommen. Fast zwangsläufig werden wohl deshalb ältere Bauten mehrheitlich positiv konnotiert, wohingegen jüngere Bauten in der Regel negativ bewertet werden.

Ein Vergleich der beiden Quartiere zeigt - neben dem bereits dargestellten quantitativen Quartierbildeffekt - ei- nen weiteren interessanten Unterschied. So wird das Länggassequartier signifikant stärker in bezug auf Komplexität differenziert ${ }^{5}(t=2.7 ;$ df: $48 ; p<.01)$, wogegen Bümpliz signifikant stärker in der Urbanität differenziert wird $(t=2.5$; df: 48; $p<.05)$. Typischerweise ist die Differenzierung der Urbanität in jenem Quartier ausgeprägter, welches im alltäglichen, sozial geteilten Quartierimage (RELPH 1976) vor allem mit «Wohnsilos» in Verbindung gebracht wird. Bereits weiter oben konnten wir zudem feststellen, daß die Bewohner dieses, in der Öffentlichkeit als "Hochhaussiedlung" stigmatisierten Quartiers die Urbanität ihres Quartiers selbst wesentlich schwächer gewichten als die Bewohner der Länggasse $(\mathrm{F}=92.1 ; \mathrm{df}: 1 / 48 ; \mathrm{p}<.001)$.

Es fällt auf, daß die Bedeutungsdimensionen der beiden Quartiere vor allem an visuellen, allgemein verfügbaren und nicht an persönlichen Gesichtspunkten sowie individuellen Erfahrungen orientiert sind. Dies hängt sicher damit zusammen, daß die hier berichteten Ergebnisse im wesentlichen die sozial geteilten, überindividuellen kognitiven Grundlagen der Quartierbilder wiedergeben. Zwei Vpn, von denen diese überindividuellen Bedeutungsdimensionen offensichtlich nicht verwendet worden sind, sollen exemplarisch für die Existenz individueller Quartierbilder evident machen, die hier nicht systematisch gruppiert und miteinander in Beziehung gesetzt werden sollen. Ihre Auswahl erfolgte aufgrund der neutralen Ausprägung (um den Nullpunkt) in den beiden überindividuellen Bedeutungsdimensionen der Quartierbilder.

\subsubsection{Zwei Beispiele individueller Bedeutungs- dimensionen von Quartieren}

Eine erste Vp hat fünf Klassen gebildet und ihren Entscheid mit den folgenden Kommentaren begründet:

1) Also diese Fotos, die ich auf diesem Haufen habe, zeigen Orte, an denen ich mich sehr gerne aufhalte. Da ist ganz verschiedenes: Wald, Bümplizer Kirche, der Jäger, eben der Stall meiner Freundin natürlich, das Heim und Hobby, das Weyerli und das Schloß Bümpliz. Dort bin ich einfach wahnsinnig gerne. Das sind alles Sachen, die ich kenne.

2) Der zweite Haufen, das sind auch Orte, die ich kenne. Tscharni, Leuen, Brïnnen da draußen. Die gefallen mir nicht besonders, aber sie interessieren mich sehr. Ich bin noch nicht viel dort gewesen, aber ich würde eigentlich gerne mehr darïber wissen, möchte einmal dorthin gehen.

3) Das hier ist ein ganz Einsames. Das dïnkt mich einfach schrecklich «biünzlig». So ähnlich wohne ich. Irgendwie ist es mir wohl dort, und gleichzeitig hasse ich dieses «bünzlige» Jedes-Gärtlein-Abtrennen.

4) Nachher, das ist der große Haufen. Das sagt mir nichts. Ich kenne es zwar. Ich gehe aber weder gerne hin noch gerne vorbei. Es sagt mir einfach nichts. Es ist weder positiv noch negativ.

5) Und dieser Haufen: Dort gehe ich einfach gerne vorbei. Ich bin nicht unbedingt gerne dort, aber ich sehe es gerne, wenn ich vorbeifahre oder vorbeilaufe. Dann freue ich mich eigentlich immer über den Anblick, den es hier bietet. 
Die nächste $V p$ hat acht Klassen gebildet, wovon fünf wiedergegeben werden:

1) Der erste Haufen ist der größte. Das sind eigentlich alles Sachen: Häuser, Strassenabschnitte, wo ich alltäglich oder praktisch jeden Tag damit zu tun habe, konkret engagiert bin, wo ich manchmal Zeug einkaufen gehe, das Staatsarchiv, wo ich eine Zeitlang gearbeitet habe fiir eine Arbeit, das Chemische Institut, welches man schon lange begrïnen sollte usw. Also es sind alles Sachen, auch die Schanze, wo ich viel mit dem Bus durchfahre, wo ich eigentlich so Alltagseindrücke davon habe. Also zu jedem Bild habe ich eine kleine Geschichte. Hier zu diesem war einmal eine kleine Schreibmaschine im Schaufenster gewesen, die angeschrieben gewesen ist, so "Hermes Baby zu verkaufen». Da bin ich dem nachgegangen, und dann ist sie leider nicht zu verkaufen gewesen. Nur so als Beispiel. Das ist so der erste Haufen.

2) Der zweite Haufen ist viel kleiner. Das sind auch Orte, die mir irgendwie wichtig sind, und zwar in dem Sinn, daß sie mir etwas sagen - vorallem in der Freizeit. Also der Bremgartenwald, wo ich vielmals laufen gehe. Hier also, ich weiß nicht, ob es der Distelweg ist, das ist so das Quartier mit den Wohnstraßen, wo wir manchmal am Abend noch spazieren gehen. Mit diesem Schulhaus assoziiere ich eine Bekannte, die da nebenan wohnt und die ich letzthin besuchte.

3) Dann der nächste Haufen ist fast ähnlich wie der, den wir gehabt haben. Nur habe ich hier einfach mehr Distanz dazu. Das scheinen mir auch Orte, wo ich gerne, aber seltener durchlaufe. Also manchmal im Sommer, wenn es schönes Wetter ist, wird der Spaziergang halt länger. Dann geht es nach hinten bis ins Zebra oder ins Bierhïbeli an ein Konzert. Das sind einfach Orte, wo ich irgendwie in zweiter Priorität gerne durchgehe.

4) Und das hier, das ist das Altersheim und der Güterbahnhof. Es hat für mich im Moment sehr viel zu tun mit essen. Es ist dort in der Nähe ein neues Restaurant aufgegangen. Und darum habe ich das hier eigentlich zusammen gruppiert.

5) Ich kann es nicht einordnen. Oder es sagt mir nichts. Es liegt fïr mich zu weit weg auf eine Art. Ich habe keine Beziehung zu diesen Orten. Ich weiß nicht einmal, wo sie sind.

Diese verbalen Kommentare machen deutlich, daß beide Vpn sich in den Strukturierungen ihres Quartiers stark von ihren persönlichen Beziehungen zu den fotografierten Orten und damit von ihren individuellen Quartierbildern haben leiten lassen. Zuneigungen und Abneigungen sowie persönliche Erlebnisse und Erfahrungen werden ebenso in die Strukturierungen miteinbezogen wie $\mathrm{Zu}$ kunftsvorstellungen und -wünsche. Deshalb fällt diese Strukturierung, die stark mit individuellen Bedeutungen, die allesamt mit vergangenen, gegenwärtigen oder zukünftigen Handlungen verknüpft sind (BOESCH 1991), auch sehr persönlich aus. Die Bilder dieser Vpn lassen sich deshalb auch schlecht in die oben dargelegten, überindividuellen, sozialisierten Quartierbilder oder Images (im Sinne von RELPH 1976) einordnen.

In den persönlichen Kommentaren zeigt sich das individuelle "Handlungspotential» (BOESCH 1980), die individuellen Ziele und Erlebnisse, welche mit den spezifi- schen Orten verwoben sind. Dabei fällt besonders deutlich die wechselseitige Verstrickung von Quartier und Bewohner auf. Das individuelle Quartierbild, so schlieBen wir deshalb, repräsentiert in starkem Maße den individuellen Kultivationsprozeß eines Bewohners in seinem Quartier, wogegen sozial geteilte Quartierbilder eher das Resultat des überindividuellen Kultivationsprozesses widerspiegeln.

\section{Folgerungen für die Bau- und Quartierplanung}

Wenn man sich abschließend die Frage stellt, welche praktischen Anregungen sich für die Stadt- und Quartierplanung aus dieser Untersuchung ableiten lassen, dann könnte man dies auf die kurzgefaßte Formel bringen: nicht nur sozialisieren, sondern auch individualisieren! Bau- und Stadtplanung orientieren sich immer noch allzu häufig daran, wie sie das überindividuelle Quartierbild erhalten und schützen können. In aller Regel schützen sie so allein Quartierbilder, die sich über den Prozeß der Sozialisation herausentwickelt haben. Das ist zwar bereits ein bemerkenswerter Fortschritt (BURCKHARDT 1981). Da aber offensichtlich jeder Bewohner teilweise über ein eigenes, individuelles Quartierbild verfügt, stellt sich zwangsläufig auch die Frage, wie sich dieses Quartierbild mit dem überindividuellen harmonisch in Einklang bringen läßt. Ein so verstandener Orts- und Quartierbildschutz verlangt in erster Linie, einer "Versteinerung» eines überindividuellen Quartierbildes in einem einmal erreichten Zustand entgegenzuwirken. Bestimmte allgemeine Bedeutungsdimensionen - wie «Urbanität» und "Komplexität» - könnten ihrerseits dazu dienen, eine erfolgte Veränderungen des Quartierbildes zu prüfen.

Obwohl sicher nicht immer leicht zu entscheiden ist, wo konkrete Veränderungen möglich sind, bedarf es ihrer, will man die individuellen in die überindividuellen Ortsbilder einfließen lassen. Die mit dieser Individualisierung erhoffte Steigerung der Wohnqualität könnte sich als Vorteil für alle erweisen.

\section{Anmerkungen}

' Die Arbeit entstand mit finanzieller Unterstützung des Schweiz. Nationalfonds (Projekt-Nr. 4025-27317).

${ }^{2}$ Die Untersuchung war Teil einer größeren empirischen Studie, in deren Rahmen der Zusammenhang zwischen Ortsbindung und Freizeitmobilität untersucht worden ist (vgl. FUHRER \& KAISER 1991; 1992a; FUHRER, KAISER und STEINER im Druck; STEINER 1992). Die Auswahl der Untersuchungsorte richtete sich dabei z. T. nach Kriterien und Wünschen des Stadtplanungsamtes der Stadt Bern.

${ }^{3}$ Beispielhaft sollen hier nur einige, ausgewählte Ergebnisse dargestellt werden. Eine ausführlichere Darstellung findet sich bei MARXER (1992). 
${ }^{4}$ Unter Urbanität versteht MONHEIM (1991) das Ergebnis europäischer Stadtkultur mit relativ dichten und kompakten Städten mit hoher Einwohner- und Arbeitsplatzdichte, gemischten Baustrukturen, vitalen Zentren und Nebenzentren sowie maßstabsgerechten Verkehrswegen.

${ }^{5}$ Untersucht werden die Varianzunterschiede in den Komplexitäts- und Urbanitätsurteilen für beide Quartiere durch dieselben Vpn.

\section{Literatur}

BARKER, R. G. (1968): Ecological psychology. Stanford, CA. BOESCH, E. E. (1980): Kultur und Handlung. Bern.

BOESCH, E.E. (1991): Symbolic action theory and cultural psychology. Berlin.

BURCKHARDT, L. (1981): Kann man das Ortsbild schützen? In: DOCU Bulletin, 6/7, 5-12.

CRANACH, M. von (1991): The multi-level organisation of knowledge and action - An integration of complexity. In: M. von CRANACH, W. DOISE und G. MUGNY (Eds.), Social representations and the social bases of knowledge. Bern.

FRIEDRICHS, J. (1977): Stadtanalyse. Soziale und räumliche Organisation der Gesellschaft. Opladen.

FUHRER, U. (1990): Handeln-Lernen im Alltag. Bern.

FUHRER, U.(1992): Behavior Settings - Mensch-Umwelt-Systeme als externalisierte soziale und kulturelle Gedächtnisse: Implikationen für eine kindergerechte Wohn- und Siedlungsgestaltung. Gastvortrag an der interdisziplinären Tagung zu kindorientierter Stadtplanung "Stadt als Rahmen kindlicher Entwicklung", Herten, 5.-9. Juli 1992.

FUHRER, U. (im Druck): Living in our own footsteps - and in those of others: Cultivation as inventive coping. Schweizerische Zeitschrift für Psychologie.

FUHRER, U., KAISER, F.G. (1991): Ortsbindung und Verkehrsdichte: Ortsbindung im Lichte räumlich-sozialer und individueller Merkmale. In: Zeitschrift für experimentelle und angewandte Psychologie, 3, 365-378.

FUHRER, U., KAISER, F. G. (1992a): Bindung an das Zuhause: die emotionalen Grundlagen. In:Zeitschrift für Sozialpsychologie, 2, 105-118.

FUHRER, U., KAISER, F. G. (1992b): Ortsbindung: Ursachen und deren Implikationen für die Wohnungs- und Siedlungsplanung. In: H.-J. HARLOFF und E. LAAGE (Hrsg.), Psychologie im Dienste der Architektur und Siedlungsplanung. Göttingen, 57-74.

FUHRER, U., KAISER, F. G., STEINER, J. (im Druck): Auto-Mobile Freizeit:Ursachen und Auswege aus der Sicht einer Psychologie des Wohnens. In: U. FUHRER und R. MEIER (Hrsg.), Wenn Autos Wohnen erst möglich machen. Zürich.

GOTTDIENER, M., LAGOPOULOS, A. Ph. (1986): The city and the sign: An introduction to urban semiotics. New York.

HART, G. (1987): "Bewußtseinsräume»: Interpretationen zu geographischen Versuchen, regionales Bewußtsein zu erforschen. In: Geographische Zeitschrift, 3, 127-148.

KUEHNE, W. (1976): Einführung in die multidimensionale Skalierung. München.
LALLI, M. (1989): Stadtbezogene Identität. Theoretische Präzisierungen und empirische Operationalisierung. In: Forschungsbericht des Institutes für Psychologie der Universität Darmstadt, Nr. 1.

LANG, A. (1992): On the knowledge in things and places. In: M. VON CRANACH, W. DOISE und G. MUGNY (Eds.), Social representations and the social bases of knowledge. Bern, 112-119.

LEWIN, K. (1917): Kriegslandschaft. In: Zeitschrift für angewandte Psychologie, 12, 440-447.

LYNCH, K. (1960): The Image of the City. Cambridge.

MARXER, M. (1992): Wie Bewohnerlnnen das Quartier «sehen". Lizentiatsarbeit. Psychologisches Institut der Universität Bern.

MUCHOW, M., MUCHOW, H. (1935): Der Lebensraum des Großstadtkindes. Darmstadt.

MONHEIM, H. (1991): Auto und Urbanität. Analyse einer Fehlentwicklung. In: T. KOENIGS und R. SCHAFFER (Hrsg.), FortSchritt vom Auto. München.

OERTER, R. (1983): Emotion als Komponente des Gegenstandsbezugs. In: H. MANDL und G. L. HUBER (Hrsg.), Emotion und Kognition. München, 282-315.

PIAGET, J. (1973): Einführung in die genetische Erkenntnistheorie. Frankfurt.

PROSHANSKY, H. M., FABIAN, A. K., KAMINOFF, R. (1983): Place-Identity: Physical World Socialization of the Self. In: Journal of Environmental Psychology, 3, 57-83.

RELPH, E. (1976): Place and placelessness. London.

ROCHBERG-HALTON, E. (1986): Meaning and modernity. Chicago.

RUSSELL, J. A., WARD, L. M., PRATT, G. (1981): Affective quality attributed to environments. In: Environment and Behavior, $13,259-286$.

SCHNEIDER, (1990): Umweltrepräsentation: Problembereiche. In: L. KRUSE, C.-F.

GRAUMANN und E.-D. LANTERMANN (Hrsg.), Ökologische Psychologie: Ein Handbuch in Schlüsselbegriffen. München, 263-267.

SIMMEL, G. (1908): Vom Wesen der Kultur. In M. LANDMANN und M. SUSMAN (Hrsg.), Brücke und Tür. Stuttgart.

STEINER, J. (1992): Bedrohte Zwischenräume - befreite Automobilisten: Freizeitmobilität aus der Optik des Wohnens. Diplomarbeit. Geographisches Institut der Universität Bern.

STOKOLS, D. (1981): Group x place transactions: Some neglected issues in psychological research on settings. In: D. MAGNUSSON (Ed.), Toward a psychology of situations. Hillsdale.

WALTHER, P. (1988): Vergleichende Studien zur Konstruktion von Bildern über Umweltwandel in Wissenschaft und Alltag. In: Landschaft und Stadt, 20(1), 1-9.

WARD, L. M., RUSSELL, J. A. (1981): The psychological representation of molar physical environments. In: Journal of Experimental Psychology: General 110, 121-152.

WEICHHARDT, P. (1989): Die Stadt Salzburg in der Vorstellung ihrer Bewohner. In: GW-Unterricht, Nr. 33, 1-18.

WEICHHARDT, P. (1990): Raumbezogene Identität. Stuttgart. 